\title{
Association of the Lung Immune Prognostic Index with Immunotherapy Outcomes in Mismatch Repair Deficient Tumors
}

\author{
Edouard Auclin ${ }^{1,+}$, Perrine Vuagnat ${ }^{2,+}$, Cristina Smolenschi ${ }^{2}$, Julien Taieb ${ }^{1}{ }^{(0}$, Jorge Adeva ${ }^{3}(\mathbb{D}$, \\ Laetitia Nebot-Bral ${ }^{4}{ }^{\text {(DD, }}$, Marta Garcia de Herreros ${ }^{6}$, Rosario Vidal Tocino ${ }^{7}$, Federico Longo-Muñoz ${ }^{8}$, \\ Yola El Dakdouki ${ }^{2}$, Patricia Martín-Romano ${ }^{2}$, Lydia Gaba 6,9 ${ }^{(1 D}$, Tamara Saurí 6,9 , Helena Oliveres ${ }^{6,9}$, \\ Eduardo Castañón ${ }^{10}$, Rocio Garcia-Carbonero ${ }^{3}$, Benjamin Besse ${ }^{11}$, Christophe Massard ${ }^{2}$, Laura Mezquita ${ }^{6,9,11, *, \ddagger}$ \\ and Antoine Hollebecque ${ }^{2, \ddagger(D)}$
}

Citation: Auclin, E.; Vuagnat, P.; Smolenschi, C.; Taieb, J.; Adeva, J.; Nebot-Bral, L.; Garcia de Herreros, M.; Vidal Tocino, R.; Longo-Muñoz, F.; El Dakdouki, Y.; et al. Association of the Lung Immune Prognostic Index with Immunotherapy Outcomes in Mismatch Repair Deficient Tumors. Cancers 2021, 13, 3776. https:// doi.org/10.3390/cancers13153776

Academic Editor: Constantin N. Baxevanis

Received: 7 June 2021

Accepted: 21 July 2021

Published: 27 July 2021

Publisher's Note: MDPI stays neutral with regard to jurisdictional claims in published maps and institutional affiliations.

Copyright: (c) 2021 by the authors. Licensee MDPI, Basel, Switzerland. This article is an open access article distributed under the terms and conditions of the Creative Commons Attribution (CC BY) license (https:/ / creativecommons.org/licenses/by/ $4.0 /)$.
1 Gastrointestinal and Medical Oncology Department, Hôpital Européen Georges Pompidou, Université de Paris, 75015 Paris, France; edouard.auclin@aphp.fr (E.A.); jtaieb75@gmail.com (J.T.)

2 Early Drug Development Department, Institut Gustave Roussy, 94805 Villejuif, France; vuagnatperrine@gmail.com (P.V.); cristina.smolenschi@gustaveroussy.fr (C.S.); yoladakdouki@hotmail.com (Y.E.D.); Patricia.MARTIN-ROMANO@gustaveroussy.fr (P.M.-R.); Christophe.MASSARD@gustaveroussy.fr (C.M.); antoine.hollebecque@gustaveroussy.fr (A.H.)

3 Medical Oncology Department, Hospital Universitario 12 de Octubre, Imas 12, UCM, 28041 Madrid, Spain; jorge.adeva@salud.madrid.org (J.A.); rgcarbonero@gmail.com (R.G.-C.)

4 UMR9019 Genome Integrity and Cancers, Gustave Roussy Cancer Campus, 94805 Villejuif, France; laetitia.nebot-bral@gustaveroussy.fr

5 Paris Saclay, Paris Sud University Orsay, 91400 Orsay, France

6 Medical Oncology Department, Hospital Clinic of Barcelona, 08036 Barcelona, Spain; garciadehe@clinic.cat (M.G.d.H.); lgaba@clinic.cat (L.G.); sauri@clinic.cat (T.S.); oliveres@clinic.cat (H.O.)

7 Medical Oncology Department, Hospital Universitario de Salamanca, IBSAL, 37007 Salamanca, Spain; mrvidal@saludcastillayleon.es

8 Medical Oncology Department, Hospital Universitario Ramon y Cajal, IRYCIS, CIBERONC, 28034 Madrid, Spain; fedelongomunoz@hotmail.com

9 Translational Genomics and Targeted Therapies in Solid Tumors, IDIBAPS, 08036 Barcelona, Spain

10 Department of Oncology, CUN-Madrid, 28027 Madrid, Spain; ecastanon@unav.es

11 Medical Oncology, Institut Gustave Roussy, 94805 Villejuif, France; benjamin.besse@gustaveroussy.fr

* Correspondence: lmezquita@clinic.cat; Tel.: +34-932275400 (ext. 380766)

+ These authors contributed equally to the work.

$\ddagger$ These authors contributed equally to the work.

Simple Summary: Deficient Mismatch Repair (dMMR) is an oncogenic path accounting for around $15 \%$ of cancers. It is considered as the first predictive marker of efficacy for immune checkpoint inhibitors (ICI). However, around 39\% of cases are refractory and additional biomarkers are needed. The Lung Immune Prognostic Index (LIPI) is a score reflecting the host inflammation, based on lactate deshydrogenase level, and derived neutrophils to leucocytes ratio. We aimed to assess the LIPI as a prognostic factor for ICI efficacy in patients with dMMR tumors. We found that patients with a Poor LIPI were more likely to experience disease progression, fast progression (death within the first 3 months of ICI), and have shorter overall and progression free survivals. This score is a low-cost, simple, and accessible prognostic tool in dMMR that merits further investigation in prospective studies.

Abstract: Background: MSI-H/dMMR is considered the first predictive marker of efficacy for immune checkpoint inhibitors (ICIs). However, around 39\% of cases are refractory and additional biomarkers are needed. We explored the prognostic value of pretreatment LIPI in MSI-H/dMMR patients treated with ICIs, including identification of fast-progressors. Methods: A multicenter retrospective study of patients with metastatic MSI-H/dMMR tumors treated with ICIs between April 2014 and May 2019 was performed. LIPI was calculated based on dNLR > 3 and LDH > upper limit of normal. LIPI groups were good (zero factors), intermediate (one factor) and poor (two factors). The primary endpoint was overall survival (OS), including the fast-progressor rate (OS < 3 months). 
Results: A total of 151 patients were analyzed, mainly female (59\%), with median age 64 years, performance status (PS) 0 (42\%), and sporadic dMMR status (68\%). ICIs were administered as first or second-line for $59 \%$. The most frequent tumor types were gastrointestinal $(66 \%)$ and gynecologic $(22 \%)$. LIPI groups were good $(47 \%)$, intermediate $(43 \%)$, and poor $(10 \%)$. The median follow-up was 32 months. One-year OS rates were $81.0 \%, 67.1 \%$, and $21.4 \%$ for good, intermediate, and poorrisk groups $(p<0.0001)$. After adjustment for tumor site, metastatic sites and PS, LIPI remained independently associated with OS (HR, poor-LIPI: 3.50, 95\%CI: $1.46-8.40, p=0.02$. Overall, the fast-progressor rate was $16.0 \%$, and $35.7 \%$ with poor-LIPI vs. $7.5 \%$ in the good-LIPI group ( $p=0.02$ ). Conclusions: LIPI identifies dMMR patients who do not benefit from ICI treatment, particularly fast-progressors. LIPI should be included as a stratification factor for future trials.

Keywords: LIPI; dNLR; LDH; MSI-H; dMMR; immunotherapy; immune checkpoint inhibitors

\section{Introduction}

Microsatellites are DNA sequences composed of short nucleotide segments which repeat sequentially. Because of their repetitive nature, these microsatellites are prone to DNA polymerase pausing and slippage during replication. In normal tissue, the mismatch repair (MMR) system usually corrects these errors [1]. However, this process may malfunction due to a constitutional or sporadic mutation in one of the repair proteins. In this case, mismatches and insertions/deletions of nucleotides accumulate in the microsatellites, resulting, first, in punctual or frameshift mutations and, second, in the activation of oncogenic genes and hypermutated phenotypes, known as microsatellite instability (MSI) or MMR deficiency (dMMR).

dMMR were initially described in colorectal cancer (CRC), reported to be involved in around $15 \%$ of tumors and could be related to Lynch syndrome, an autosomal dominant cancer-predisposition syndrome caused by mutations in MMR strains [2]. However, MSI can also be observed in sporadic CRC, which is more likely related to the epigenetic inactivation of the MLH1 gene expression promoter. MSI has also been observed in other tumor types ( $1 \%$ to $30 \%$ depending on the histological type), with endometrial carcinoma and gastric cancer being the most common $[3,4]$.

MSI-H and dMMR, both early conditions of hypermutability, increase the generation of neoantigens leading to increased tumor immunogenicity and high tumor mutational burden [5], suggesting that immunotherapy could be an interesting approach in patients harboring these mutations. Research led to dMMR receiving FDA-approval as the first agnostic tumor-related predictive biomarker for PD-1 inhibitors. In a cohort of patients with MSI tumors, pembrolizumab demonstrated response in between $40-70 \%$ of patients vs. none in microsatellite stable tumors [6]. Following this trial, other studies have demonstrated similar findings with other PD-1 inhibitors [7-10].

Despite the impressive data reported, not all patients with dMMR tumors respond to PD-1 inhibitors, and there is a need for additional biomarkers for immune checkpoint inhibitors (ICIs). Host-related biomarkers are gaining importance in immune-oncology, providing additional information from the patient (host) that can be integrated into immunotherapy outcome prediction. Among them, the lung immune prognostic index (LIPI), based on two inflammatory parameters (derived neutrophil to leucocytes ratio [dNLR] calculated as neutrophil count/[leucocytes count-neutrophils count], and lactate dehydrogenase [LDH]) correlated strongly with immunotherapy outcomes in large cohorts of patients with non-small cell lung cancer, and in other tumor types such as renal and head and neck cancers [11-15].

In this study, we aimed to assess the prognostic value of LIPI in a large multicenter cohort of patients treated with immunotherapy for dMMR tumors, and whether LIPI can identify fast progressors who are undergoing immunotherapy. 


\section{Materials and Methods}

\subsection{Study Design and Patients}

This is a retrospective multicenter study of patients with advanced solid tumors with dMMR status, treated with anti-PD-1 or PD-L1 ICIs (atezolizumab, avelumab, nivolumab +/ - ipilimumab, pembrolizumab, and other phase I drugs) in a variety of settings including routine clinical care, expanded access programs, and clinical trials, between April 2014 and May 2020 across seven European tertiary referral hospitals in France and Spain. We collected baseline clinical, pathological, and biological data prior to immunotherapy, including leucocyte and neutrophil counts, $\mathrm{LDH}$, and albumin. Radiological assessment was performed every 8-12 weeks according to local practice and evaluated per Response Evaluation Criteria in Solid Tumors v1.1 (RECIST). The study was approved by the Institutional Review Board of the Institut Gustave Roussy on 18th March 2021 (Registration Number 2021-26). Informed consent was not required for this retrospective study.

\subsection{Lung Immune Prognostic Index}

The LIPI was calculated based on dNLR $>3$ (one point) and LDH $>$ upper limit of normal (ULN) (one point), as previously reported [11]. Patients with no points were classified in the good group, patients with 1 point (dNLR $>3$ or LDH $>$ ULN) in the intermediate group, and those with 2 points (dNLR $>3$ and LDH $>\mathrm{ULN}$ ) in the poor group.

\subsection{MSI-H/dMMR Status}

MMR status was analyzed according to the techniques used in each center, including immunohistochemistry, polymerase chain reaction (PCR), next-generation, or whole exome sequencing.

\subsection{Statistical Analysis}

Median (IQR) values and proportions (percentage) were used for continuous and categorical variables, respectively. Median and proportions were compared using the Wilcoxon-Mann-Whitney test and the chi ${ }^{2}$ test (or Fisher's exact test, if appropriate), respectively. The primary endpoint was overall survival (OS) defined as the time between the start of ICI treatment and death from any cause. The other primary endpoint was the fast progressors rate (FPR), defined as an OS $<3$ months. Secondary endpoints were progression-free survival (PFS), ORR, and disease control rate (DCR). PFS was defined as the time between ICI start and progressive disease (PD) or death, whichever occurred first. ORR was defined as the sum of complete and partial responses according to RECIST 1.1. DCR was defined as the sum of complete and partial responses and stable disease according to RECIST 1.1. OS and PFS were estimated using the Kaplan Meier method, and groups were compared with the log-rank test. Follow-up was calculated using the reverse Kaplan Meier method. The association of demographic, clinical, and biological factors with survival was assessed with univariate and multivariate Cox proportional-hazard models, providing a hazard ratio (HR) and its $95 \%$ confidence interval (CI). The association between LIPI and FPR ORR and DCR was evaluated with logistic regression, providing an odds ratio (OR) and its 95\%CI. All analyses were performed using R software version 2.15.2 ( $\mathrm{R}$ Development Core Team, Vienna, Austria). $p$ values $<0.05$ were considered statistically significant, and all tests were two-sided.

\section{Results}

\subsection{Study Population}

A total of 151 patients were included with a median follow-up of 32.1 months $(95 \% \mathrm{CI}$ 24.8-36.3).

The main baseline characteristics are summarized in Table 1 . The most common tumor types were gastrointestinal $(65.6 \% ; 60.6 \%$ CRC, $39.4 \%$ others) followed by gynecologic tumors (21.8\%) (Figure S1). In the 146 patients with the available data, dMMR status was diagnosed by PCR alone in 5 patients (3.4\%), immunohistochemistry and PCR in 135 pa- 
tients $(92.5 \%)$, with next-generation or whole exome sequencing (alone or in combination with the other techniques) in 11 patients $(7.5 \%)$. dMMR status was associated with Lynch syndrome in $40(32.0 \%)$ patients ( $80.0 \%$ CRC, $5.0 \%$ gynecologic, $15.0 \%$ other).

Table 1. Clinical, pathological and biological characteristics of the population.

\begin{tabular}{|c|c|c|}
\hline Variable & & $\begin{array}{l}\text { All Patients } \\
(\mathbf{N}=151)\end{array}$ \\
\hline \multirow[t]{3}{*}{ Age } & Median (IQR) & $64(51.5-70.5)$ \\
\hline & $>65$ & $64(43.0 \%)$ \\
\hline & Missing & 2 \\
\hline \multirow[t]{2}{*}{ Gender } & female & $89(58.9 \%)$ \\
\hline & male & $62(41.1 \%)$ \\
\hline \multirow[t]{3}{*}{ Primary tumor site } & gastrointestinal & $99(65.6 \%)$ \\
\hline & gynecologic & $33(21.8 \%)$ \\
\hline & other & $19(12.6 \%)$ \\
\hline \multirow[t]{2}{*}{ Lynch syndrome } & yes & $40(32.0 \%)$ \\
\hline & missing & 26 \\
\hline \multirow[t]{3}{*}{ Line of ICI start } & median (IQR) & $2(2-3)$ \\
\hline & $>2$ & $62(41.3 \%)$ \\
\hline & missing & 1 \\
\hline \multirow{2}{*}{$\begin{array}{c}\text { Number of metastasis at ICI } \\
\text { start }\end{array}$} & $>2$ & $34(23.6 \%)$ \\
\hline & missing & 7 \\
\hline \multirow[t]{4}{*}{ Metastasis sites } & lung metastasis & $31(20.5 \%)$ \\
\hline & bone metastasis & $13(8.6 \%)$ \\
\hline & liver metastasis & $47(31.1 \%)$ \\
\hline & brain metastasis & $7(4.6 \%)$ \\
\hline \multirow[t]{2}{*}{ Type of ICI antibody } & PD-1 & $109(72.2 \%)$ \\
\hline & PD-L1 & $42(27.8 \%)$ \\
\hline \multirow[t]{2}{*}{ Monotherapy or combination } & combination & $20(13.3 \%)$ \\
\hline & monotherapy & $131(86.7 \%)$ \\
\hline \multirow[t]{3}{*}{ Performance status at ICI start } & 0 & $58(42.3 \%)$ \\
\hline & $\geq 1$ & $79(57.7 \%)$ \\
\hline & missing & 14 \\
\hline dNLR & $>3$ & $39(25.8 \%)$ \\
\hline \multirow[t]{2}{*}{$\mathrm{LDH}$} & high & $55(38.5 \%)$ \\
\hline & missing & 8 \\
\hline \multirow[t]{2}{*}{ Albumin $(\mathrm{g} / \mathrm{L})$} & $\leq 35$ & $64(43.5 \%)$ \\
\hline & missing & 4 \\
\hline
\end{tabular}

ICI: immune checkpoint inhibitors, dNLR: derived neutrophils to leukocytes ratio, LDH: lactate dehydrogenase, LIPI: lung immune prognostic index.

Median OS was not reached (NR) in the overall population, (95\%CI: 23.4 to NR), and the 1-year OS rate was $69.3 \%$ (95\%CI: 62.0 to 77.6). Median PFS was 10.5 months (95\%CI: 7.1 to 35.1 ) and the 1-year PFS rate was $47.8 \%$ (95\%CI: 40.1 to 56.9) (Table 2).

\subsection{LIPI in dMMR Tumors}

Pretreatment median dNLR was 2.29 (interquartile range, IQR: 1.61-3.09) and was $>3$ in $25.8 \%$ of patients. Pretreatment median LDH was 218 IU/L (IQR: 188.5-313) and was $>$ ULN in 38.5\%. Considering both parameters, LIPI was evaluable in 143 patients, and classified the population into three prognostic groups: good $(n=67,46.9 \%)$, intermediate $(n=62,43.3 \%)$ and poor $(n=14,9.8 \%)$ (Figure S2). The baseline characteristics of the population by LIPI group $(n=143)$ are summarized in Tables S1 and S2. The presence of 
brain metastasis, high number of metastatic sites ( $>2)$, poor PS and hypoalbuminemia at ICI start were associated with poor LIPI.

Table 2. Median survival and response endpoints according to the LIPI score.

\begin{tabular}{|c|c|c|c|c|c|c|}
\hline Variable & & $\begin{array}{l}\text { All Patients } \\
(\mathbf{N}=151)\end{array}$ & $\begin{array}{c}\text { LIPI Good } \\
\text { Prognostic Group } \\
(\mathrm{N}=67)\end{array}$ & $\begin{array}{l}\text { LIPI Intermediate } \\
\text { Prognostic Group } \\
\qquad(\mathrm{N}=62)\end{array}$ & $\begin{array}{c}\text { LIPI Poor } \\
\text { Prognostic Group } \\
(\mathrm{N}=14)\end{array}$ & $p$ \\
\hline \multirow{2}{*}{ Median (95\%CI) } & OS & NR (23.4 to NR) & NR (36.5 to NR) & NR (16.2 to NR) & 3.3 (2.6 to NR) & $<0.001$ \\
\hline & PFS & $10.5(7.1$ to 35.1$)$ & $20.9(8.4$ to $\mathrm{NR})$ & $9.9(2.8$ to $\mathrm{NR})$ & $2.3(1.8$ to NR) & $<0.001$ \\
\hline \multirow{3}{*}{$\begin{array}{l}\text { Fast progressors } \\
\text { rate }\end{array}$} & yes & $24(16.0 \%)$ & $5(7.5 \%)$ & $11(18.0 \%)$ & $5(35.7 \%)$ & 0.02 \\
\hline & no & $126(84.0 \%)$ & $62(92.5 \%)$ & $50(82.0 \%)$ & $9(64.3 \%)$ & \\
\hline & missing & 1 & 0 & 1 & 0 & \\
\hline \multirow{3}{*}{ ORR } & no & $87(60.8 \%)$ & $35(53.8 \%)$ & $38(64.4 \%)$ & $11(91.7 \%)$ & 0.03 \\
\hline & yes & $56(39.2 \%)$ & $30(46.2 \%)$ & $21(35.6 \%)$ & $1(8.3 \%)$ & \\
\hline & missing & 8 & 2 & 3 & 2 & \\
\hline \multirow{3}{*}{ DCR } & no & $47(32.6 \%)$ & $10(15.2 \%)$ & $26(44.1 \%)$ & $9(75.0 \%)$ & $<0.001$ \\
\hline & yes & $97(67.4 \%)$ & $56(84.8 \%)$ & $33(55.9 \%)$ & $3(25.0 \%)$ & \\
\hline & missing & 7 & 1 & 3 & 2 & \\
\hline
\end{tabular}

95\%CI: 95\% confidence interval, ORR: objective response rate, DCR: disease control rate.

\subsection{LIPI Is Associated with ICI Survival Outcomes in MSI-H Tumors}

LIPI was associated with both OS and PFS $(p<0.0001)$. Median OS was NR $(95 \% \mathrm{CI}$ 36.5 to NR), NR (95\%CI 16.2 to NR), and 3.3 months (95\%CI 2.6 to NR) for the good, intermediate, and poor LIPI groups, respectively $(p<0.001)$ (Figure $1 \mathrm{~A}$, Table 2$)$. The one-year OS rates for good, intermediate, and poor-LIPI groups were $81.0 \%(95 \%$ CI 71.5 to $91.9), 67.1 \%$ (95\%CI 56.0 to 80.5$)$, and $21.4 \%$ (95\%CI 7.9 to 58.4$)$, respectively $(p<0.001)$.

A

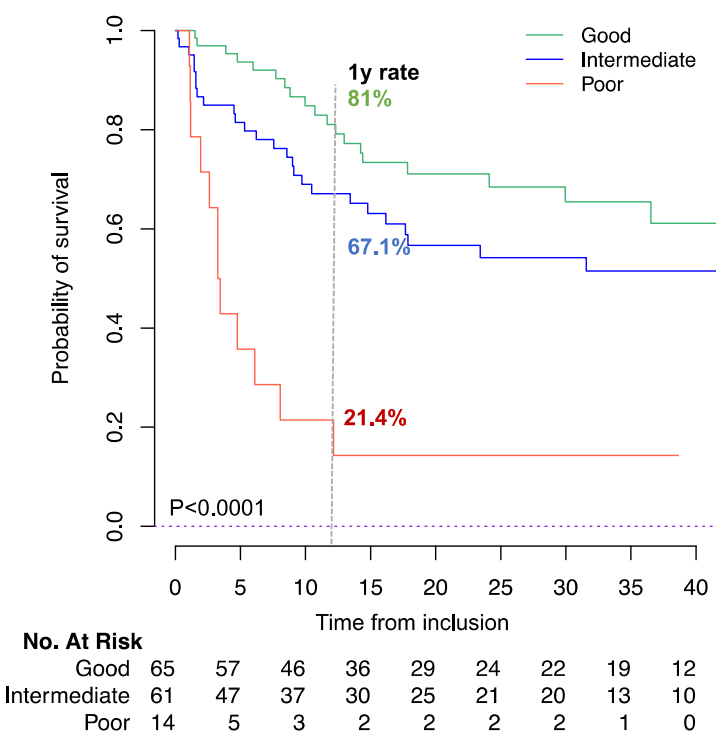

B

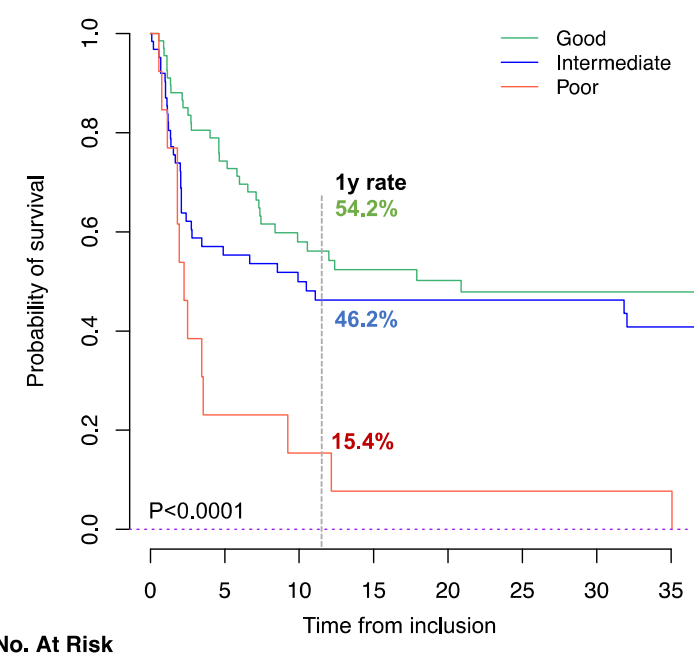

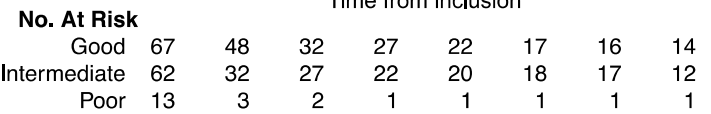

Figure 1. Kaplan Meier curve for OS (A) and PFS (B) according to LIPI score. Log Rank $p<0.0001$ for both endpoints. The numbers at risk differ between OS and PFS, due to missing data in OS/PFS status or duration.

Similarly, median PFS was 20.9 months (95\%CI 8.4 to NR), 9.9 months (95\%CI 2.8 to $\mathrm{NR}$ ), and 2.3 months (95\%CI 1.8 to NR) in the good, intermediate, and poor-LIPI groups, respectively $(p<0.0001$; Table 2$)$. The one-year PFS rates for good, intermediate, and poor-LIPI groups were $54.2 \%$ (95\%CI 43.1 to 68.2$), 46.2 \%$ (95\%CI 35.1 to 61.0$)$, and $15.4 \%$ (95\%CI 4.3 to 55.0$)$, respectively $(p<0.0001)$, Figure $1 \mathrm{~B}$. 
In the multivariate analysis, including tumor location, number of metastasis before ICI, ECOG PS, and albumin levels, LIPI was an independent factor for OS (HR for intermediate, 1.43 [95\%CI 0.75 to 2.74]; HR for poor, 3.50 [95\%CI 1.46 to 8.40 ], $p=0.03$ ). In terms of PFS, the HRs for the intermediate and poor groups were 1.09 (95\%CI 0.65 to 1.82 ) and 2.41 (95\%CI 1.12 to 5.19), respectively ( $p=0.07$ ) (Table 3). The c-index of LIPI for OS and PFS prediction are reported in Table S3.

Table 3. Multivariate Cox model analysis for OS and PFS in the population.

\begin{tabular}{|c|c|c|c|c|c|c|c|}
\hline \multirow[t]{2}{*}{ Variable } & & \multicolumn{3}{|c|}{$\begin{array}{c}\text { OS } \\
\mathrm{N}=\mathbf{1 2 5}, \mathrm{n} \text { Events }=49\end{array}$} & \multicolumn{3}{|c|}{$\begin{array}{c}\text { PFS } \\
\mathrm{N}=124, \mathrm{n} \text { Events }=71\end{array}$} \\
\hline & & HR & $95 \% \mathrm{CI}$ & $p$ & HR & $95 \% \mathrm{CI}$ & $p$ \\
\hline \multirow[t]{3}{*}{ Tumor site } & gastrointestinal & 1 & & & 1 & & \\
\hline & gynecologic & 1.65 & 0.82 to 3.29 & 0.03 & 1.53 & 0.84 to 2.81 & 0.0002 \\
\hline & other & 2.59 & 1.25 to 5.35 & & 4.08 & 2.08 to 8.01 & \\
\hline $\begin{array}{c}\text { N metastatic sites at ICI } \\
\text { start }\end{array}$ & $>2$ & 1.99 & 1.06 to 3.70 & 0.03 & 1.06 & 0.61 to 1.85 & 0.84 \\
\hline \multirow[t]{2}{*}{ Performance status } & 0 & & & & & & \\
\hline & $\geq 1$ & 2.11 & 1.05 to 4.24 & 0.04 & 1.91 & 1.10 to 3.31 & 0.02 \\
\hline Albumin (g/L) & $>35$ & 0.82 & 0.45 to 1.50 & 0.51 & 0.96 & 0.58 to 1.59 & 0.87 \\
\hline \multirow[t]{3}{*}{ LIPI } & good & 1 & & & 1 & & \\
\hline & tntermediate & 1.43 & 0.75 to 2.74 & 0.02 & 1.09 & 0.65 to 1.82 & 0.07 \\
\hline & poor & 3.50 & 1.46 to 8.40 & & 2.41 & 1.12 to 5.19 & \\
\hline
\end{tabular}

OS: overall survival, PFS: progression-free survival, HR: hazard ratio, 95\%CI: 95\% confidence interval, ICI: immune checkpoint inhibitors, LIPI: lung immune prognostic index.

\subsection{LIPI Is Associated with Tumor Response under ICI in dMMR Tumors}

We also studied the impact of LIPI on response outcomes. The ORR in the overall population was $39.2 \%$. According to LIPI group, the ORR was $46.2 \%$ in the good group, $35.6 \%$ in the intermediate, and $8.3 \%$ in the poor group $(p=0.03)$ (Table 2, Figure 2). Patients in the poor-LIPI group had a higher risk of experiencing nonresponse compared with the good-LIPI group, with an OR $9.43(95 \%$ CI 1.15 to $77.27, p=0.04)$ (Table 4). Similarly, PD as best response (absence of DCR), was significantly associated with the poor-LIPI group.

\subsection{Fast Progressors Rate}

We also evaluated the FPR in our dMMR population. Overall, the FPR was $16.0 \%$ $(n=24 / 150)$ (Table 2). The distribution of fast progressors was not different among the different tumor types. When considered with reference to the LIPI group, the FPR was significantly higher in the poor -LIPI group, with $35.7 \%$ of patients $(n=5 / 14)$, compared with the intermediate group with $18.0 \%(n=11 / 62)$ and the good-LIPI group with $7.5 \%$ $(n=5 / 67), p=0.02$ (Table 2). Additionally, the poor group had a significantly higher risk of experiencing fast progression compared with the good-LIPI group, with an OR of 6.89 (95\%CI 1.66 to $28.59, p=0.01$ ) (Table 4 ).

Table 4. Univariate logistic regression for response endpoints according to LIPI score.

\begin{tabular}{|c|c|c|c|c|c|c|}
\hline \multirow[t]{2}{*}{ Variable } & \multicolumn{2}{|c|}{ ORR } & \multicolumn{2}{|c|}{ DCR } & \multicolumn{2}{|c|}{ Fast Progressors } \\
\hline & OR $(95 \% \mathrm{CI})$ & $p$ & OR $(95 \% \mathrm{CI})$ & $p$ & OR $(95 \% \mathrm{CI})$ & $p$ \\
\hline \multicolumn{7}{|l|}{ LIPI } \\
\hline Good & Ref & & Ref & & Ref & \\
\hline Intermediate & $1.55(0.75-3.19)$ & 0.23 & $4.41(1.89-10.29)$ & 0.01 & $2.73(0.89-8.37)$ & 0.08 \\
\hline Poor & $9.43(1.15-77.27)$ & 0.04 & $16.8(3.86-73.05)$ & $<0.0001$ & $6.89(1.66-28.59)$ & 0.01 \\
\hline
\end{tabular}




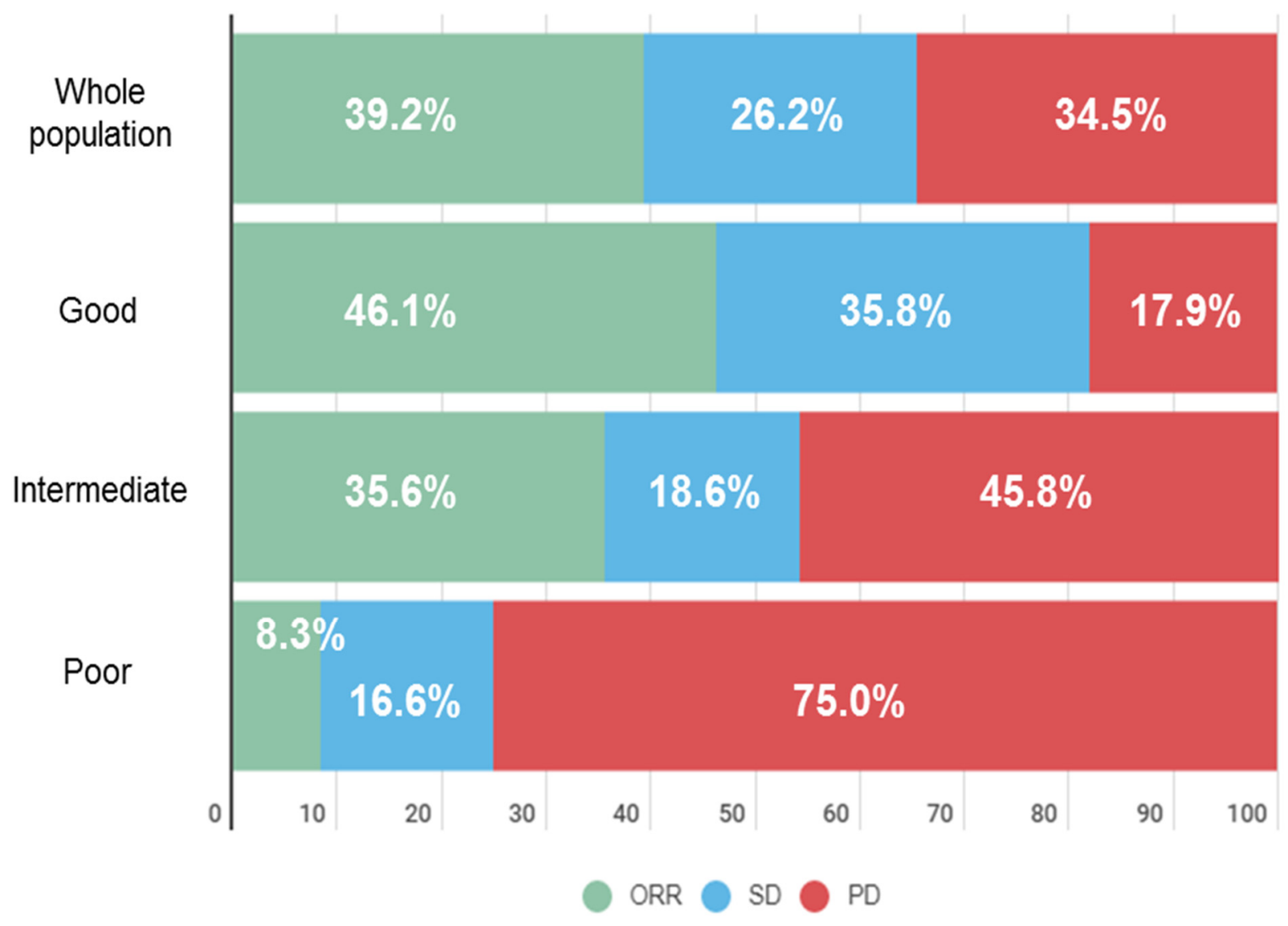

Figure 2. Best response according to LIPI group. ORR: objective response rate, SD: stable disease, PD: progression disease.

\section{Discussion}

In this study, we have shown for the first time the impact of host-related biomarkers, represented by circulating inflammatory parameters combined as the LIPI score (dNLR and LDH), on ICI outcomes in a large cohort of patients with advanced dMMR solid tumors, widely considered a favorable population for ICI. In this study, LIPI was an independent prognostic factor for OS, with the poor-LIPI group being associated with worse immunotherapy outcomes, suggesting that host-related biomarkers are also relevant for this population. This poor-LIPI group comprised a subset of patients $(9.8 \%$ of the patients) with no clear benefit for immunotherapy, despite of their dMMR status, with a median OS of 3.3 months, median PFS of 2.3 months, and a 35.7\% rate of fast-progressors.

Interestingly, we observed in our population, composed primarily of gastrointestinal and gynecologic tumors, similar data as previously reported in other tumor types, notably NSCLC [11-15], suggesting that these parameters reflect the host immune context regardless of the tumor type and other tumor-related biomarkers, such as dMMR. Of note in these previous studies, median OS for the evaluated poor-LIPI subgroups ranged from 2.6 to 5.0 months, and median PFS ranged from 1.2 to 2.3 months [11-16].

Inflammation and activation of the innate immune system is a well-known resistance pathway for ICIs, promoting tumor growth and dissemination [17-19]. Among the innate immune cells, neutrophils are one of the major actors. As a neutrophil-based ratio, LIPI is thus a good indicator of the circulating inflammatory status of patients before receiving ICI therapy. Systemic inflammation is capable of inducing IDO (indoleamine 2,3-dioxygenase) and also turning immune infiltration towards immunotolerance [20]. IDO plays an immunosuppressive role, preventing perpetual inflammation. IDO expression in MSI tumors is heterogeneous [21]. High IDO expression induced by systemic inflammation could 
explain the differences in response to ICIs, even in MSI patients. It has also been described that MSH3 mutations, which result in the EMAST phenotype (elevated microsatellite alterations at selected tetranucleotide repeats), could lead to chronic inflammation mediated by IL-6 and TNF-alpha [22]. These EMAST patients had shorter survival and an aggressive tumor phenotype. The good prognostic value of the LIPI score in the MSI population is explained by its capacity to reflect the patient's systemic inflammation.

dMMR is a validated, FDA-approved, tumor-based predictive biomarker of response to ICI, with impressive data in terms of response $40 \%$ to $55 \%$ in CRC with dMMR, and $34 \%$ to $71 \%$ in non-CRC tumors) [7-10]. Despite these encouraging ORRs, PD as best response can represent $12 \%$ to $61 \%$ of this population, depending on the histological subtype, and no biomarkers are available to screen these patients in order to address this [6]. In our study, we observed $32.6 \%$ of patients with refractory disease having PD as best response, however this increased significantly in the poor-LIPI group, reaching $75 \%$. This suggested that LIPI could be a useful tool to better identify true responders and, even more relevantly, to identify populations unlikely to respond to immunotherapy, providing additional information on outcome prediction, compared to the restricted vision, when using only tumor-based biomarkers. Similarly, LIPI, as for other host-related biomarkers, has also been explored in $\geq 50 \%$ PD-L1 NSCLC, another favorable population for immunotherapy [23]. In a cohort of 930 patients treated with ICIs, LIPI was an independent prognostic marker regardless of PD-L1 expression. Both this and our study have highlighted the concept that LIPI can provide additional relevant information to already-known tumor-based biomarkers (e.g., PD-L1, dMMR), and could be explored in combination with these well-established biomarkers in clinical trials. The predictive values of LIPI on ICI benefit have already been investigated in NSCLC [24,25].

In our study, we defined fast progressors as patients with an OS $<3$ months after ICI start. This is one of the aggressive progression patterns described in cancer patients under immunotherapy $[23,26]$. Although the immunological mechanisms have not been well established and this phenomenon remains controversial, some patients experience rapid and aggressive progression and previous reports highlighted inflammation as a key mechanism for the aggressive patterns [26,27]. In our study, we described $16 \%$ of our population as fast progressors, however this proportion was notably higher (35\%) in the poor-LIPI population. This could be the first clinical evidence of the link between circulating inflammatory status and refractory disease under immunotherapy. In this context, we observed that single agent ICIs are not able to overcome this primary resistance, even in a good-responder population (dMMR). In the future, LIPI could serve as a tool for clinicians to select the best treatment strategy, as is being explored in NSCLC [23-25].

Our study has a number of limitations directly related to its retrospective nature, notably missing clinical data (for example other possible interesting inflammatory biomarkers such a C-Reactive Protein). Secondly, patients were treated with various immunotherapy drugs, which can lead to heterogeneity in terms of efficacy; nevertheless, our cohort is representative of the dMMR population, with outcome data for response and survival consistent with previous data reported in the literature. Finally, the lack of other treatment cohorts with combination therapies or only chemotherapy as a comparison limited our exploration of the potential predictive rather than prognostic role of LIPI in this dMMR cohort.

Nonetheless, this study represents the first proof of concept that the patient's hostimmune context can play an important role in dMMR patients receiving immunotherapy. We demonstrated, in the largest multicenter cohort of patients with dMMR tumors reported to date, that LIPI can offer useful information on outcome prediction to the current exclusive context of tumor-based biomarkers. The integration of these host-related biomarkers with tumor-based biomarkers will improve identification of relevant data in the decision-making process for selecting the best therapeutic strategy in cancer patients. 


\section{Conclusions}

The LIPI, based on pretreatment dNLR and LDH, is associated with ICI outcomes in the MSI-H/d-MMR population, with LIPI demonstrated to be an independent factor for OS. LIPI can identify the population with higher risk of progression or death under ICIs, the poor-LIPI group with high-dNLR/high-LDH. This score is a low-cost, simple, and accessible prognostic tool in dMMR that merits further investigation in prospective studies.

Supplementary Materials: The following are available online at https:/ / www.mdpi.com/article/10 .3390 / cancers13153776/s1, Figure S1: distribution of tumor types in the study population $(n=151)$, Figure S2: distribution of the LIPI groups in the study population, Table S1: clinical, pathological and biological characteristics of the population according to LIPI group $(n=143)$, Table S2: detailed tumor types according to LIPI group $(n=143)$, Table S3: LIPI c-index for OS and PFS prediction.

Author Contributions: Study design: E.A., P.V., L.M., A.H.; Data collection: E.A., P.V., C.S., J.T., J.A., L.N.-B., M.G.d.H., R.V.T., F.L.-M., Y.E.D., P.M.-R., L.G., T.S., H.O., E.C., R.G.-C., B.B., C.M.; Manuscript writing and review: all authors; Statistical analysis: E.A. All authors have read and agreed to the published version of the manuscript.

Funding: This research did not receive any specific grant from funding agencies in the public, commercial, or not-for-profit sectors. Laura Mezquita received support from the IASLC Research Fellowship Award (2018), ESMO Translational Research Fellowship (2019) and SEOM retorno de Investigadores (2019); Contrato Juan Rodés 2020; Ayuda SEOM-Juan Rodés 2020.

Institutional Review Board Statement: This study was approved by the Ethics committee of the Institut Gustave Roussy on 18th March 2021 (Registration Number 2021-26).

Informed Consent Statement: No consent to participate was needed for this retrospective study.

Data Availability Statement: Contact the corresponding author.

Conflicts of Interest: E. Auclin: Travel/Accommodation/Expenses: Mundipharma; Honoraria (self): Sanofi Genzymes. J. Taieb: Honoraria (self): Merck, Roche, Amgen, Lilly, Sanofi, Samsung, MSD, Servier, Celgene, Pierre Fabre; Advisory/Consultancy: Roche, Merck KGaA, Amgen, Lilly, MSD, Servier, Pierre Fabre, Sanofi, Samsung; Speaker Bureau/Expert testimony: Servier, Amgen, Roche, Sanofi, Merck, Lilly, Pierre Fabre. B. Besse: sponsored research at Gustave Roussy Cancer Center 4D Pharma, Abbvie, Amgen, Aptitude Health, AstraZeneca, BeiGene, Blueprint Medicines, BMS, Boehringer Ingelheim, Celgene, Cergentis, Cristal Therapeutics, Daiichi-Sankyo, Eli Lilly, GSK, Inivata, Janssen, Onxeo, OSE immunotherapeutics, Pfizer, Roche-Genentech, Sanofi, Takeda, Tolero Pharmaceuticals. C. Massard: Advisory/Consultancy: Amgen, Astellas, AstraZeneca, Bayer, BeiGene, BMS, Celgene, Debiopharm, Genentech, Ipsen, Janssen, Lilly, MedImmune, MSD, Novartis, Pfizer, Roche, Sanofi, Orion. L. Mezquita: Research grant/Funding (self): Bristol Myers Squibb, Boehringer Ingelheim, Amgen, Stilla, Inivata; Advisory/Consultancy: Roche Diagnostics, Takeda; Honoraria (self): Bristol Myers Squibb, Tecnofarma, Roche; Travel/Accommodation/Expenses: Roche; Non-remunerated activity/ies: AstraZeneca. A. Hollebecque: Advisory/Consultancy: Gritstone Oncology, Eisai Co., Ltd., Amgen, Servier and Merck Serono. All other authors have declared no conflicts of interest.
Abbreviations
dMMR deficient mismatch repair
dNLR derived neutrophils/leucocytes ratio
LIPI lung immune prognostic index
MSI-H microsatellites instable high
OS overall survival
PFS progression free survival 


\section{References}

1. Cilona, M.; Locatello, L.G.; Novelli, L.; Gallo, O. The Mismatch Repair System (MMR) in Head and Neck Carcinogenesis and Its Role in Modulating the Response to Immunotherapy: A Critical Review. Cancers 2020, 12, 3006. [CrossRef] [PubMed]

2. Luchini, C.; Bibeau, F.; Ligtenberg, M.; Singh, N.; Nottegar, A.; Bosse, T.; Miller, R.; Riaz, N.; Douillard, J.-Y.; Andre, F.; et al. ESMO recommendations on microsatellite instability testing for immunotherapy in cancer, and its relationship with PD-1/PD-L1 expression and tumour mutational burden: A systematic review-based approach. Ann. Oncol. 2019, 30, 1232-1243. [CrossRef] [PubMed]

3. Deshpande, M.; Romanski, P.A.; Rosenwaks, Z.; Gerhardt, J. Gynecological Cancers Caused by Deficient Mismatch Repair and Microsatellite Instability. Cancers 2020, 12, 3319. [CrossRef] [PubMed]

4. Eso, Y.; Seno, H. Current status of treatment with immune checkpoint inhibitors for gastrointestinal, hepatobiliary, and pancreatic cancers. Ther. Adv. Gastroenterol. 2020, 13. [CrossRef] [PubMed]

5. Le, D.T.; Durham, J.N.; Smith, K.N.; Wang, H.; Bartlett, B.R.; Aulakh, L.K.; Lu, S.; Kemberling, H.; Wilt, C.; Luber, B.S.; et al. Mismatch repair deficiency predicts response of solid tumors to PD-1 blockade. Science 2017, 357, 409-413. [CrossRef] [PubMed]

6. Le, D.T.; Uram, J.N.; Wang, H.; Bartlett, B.R.; Kemberling, H.; Eyring, A.D.; Skora, A.D.; Luber, B.S.; Azad, N.S.; Laheru, D.; et al. PD-1 Blockade in Tumors with Mismatch-Repair Deficiency. N. Engl. J. Med. 2015, 372, 2509-2520. [CrossRef] [PubMed]

7. Le, D.T.; Kim, T.W.; Van Cutsem, E.; Geva, R.; Jäger, D.; Hara, H.; Burge, M.; O’Neil, B.; Kavan, P.; Yoshino, T.; et al. Phase II OpenLabel Study of Pembrolizumab in Treat-ment-Refractory, Microsatellite Instability-High/Mismatch Repair-Deficient Metastatic Colorectal Cancer: KEYNOTE-164. J. Clin. Oncol. J. Am. Soc. Clin. Oncol. 2020, 38, 11-19. [CrossRef]

8. André, T.; Shiu, K.-K.; Kim, T.W.; Jensen, B.V.; Jensen, L.H.; Punt, C.; Smith, D.; Garcia-Carbonero, R.; Benavides, M.; Gibbs, P.; et al. Pembrolizumab in Microsatellite-Instability-High Advanced Colorectal Cancer. N. Engl. J. Med. 2020, 383, $2207-2218$. [CrossRef]

9. Overman, M.J.; McDermott, R.; Leach, J.L.; Lonardi, S.; Lenz, H.-J.; Morse, M.A.; Desai, J.; Hill, A.; Axelson, M.; Moss, R.A.; et al. Nivolumab in patients with metastatic DNA mismatch repair-deficient or microsatellite instability-high colorectal cancer (CheckMate 142): An open-label, multicentre, phase 2 study. Lancet Oncol. 2017, 18, 1182-1191. [CrossRef]

10. Marabelle, A.; Le, D.T.; Ascierto, P.A.; Di Giacomo, A.M.; De Jesus-Acosta, A.; Delord, J.-P.; Geva, R.; Gottfried, M.; Penel, N.; Hansen, A.R.; et al. Efficacy of Pembrolizumab in Patients with Noncolorectal High Microsatellite Instability/Mismatch Repair-Deficient Cancer: Results From the Phase II KEY-NOTE-158 Study. J. Clin. Oncol. J. Am. Soc. Clin. Oncol. 2020, 38, 1-10. [CrossRef]

11. Mezquita, L.; Auclin, E.; Ferrara, R.; Charrier, M.; Remon, J.; Planchard, D.; Ponce, S.; Ares, L.P.; Leroy, L.; Audigier-Valette, C.; et al. Association of the Lung Immune Prognostic Index with Immune Checkpoint Inhibitor Outcomes in Patients With Advanced Non-Small Cell Lung Cancer. JAMA Oncol. 2018, 4, 351-357. [CrossRef]

12. Varga, A.; Bernard-Tessier, A.; Auclin, E.; Pérez, L.M.; Baldini, C.; Planchard, D.; Marabelle, A.; Hollebecque, A.; Besse, B.; Massard, C. Applicability of the lung immune prognostic index (LIPI) in patients with metastatic solid tumors when treated with immune checkpoint inhibitors (ICI) in early clinical trials. Ann. Oncol. 2019, 30, i2. [CrossRef]

13. Benitez, J.C.; Recondo, G.; Rassy, E.; Mezquita, L. The LIPI score and inflammatory biomarkers for selection of patients with solid tumors treated with checkpoint inhibitors. Q. J. Nucl. Med. Mol. Imaging 2020, 64. [CrossRef]

14. Lavaud, P.; Dalban, C.; Negrier, S.; Chevreau, C.; Gravis, G.; Oudard, S.; Laguerre, B.; Barthelemy, P.; Borchiellini, D.; Goupil, M.G.; et al. Validation of the lung immune prognostic index (LIPI) in patients with metastatic renal cell carcinoma treated with nivolumab in the GETUG-AFU 26 NIVOREN trial. J. Clin. Oncol. 2020, 38, 735. [CrossRef]

15. Chen, M.; You, R.; You-Ping, L.; Huang, P.-Y.; Zou, X.; Shen, G.-P.; Zhang, H.-D. Chemotherapy plus local-regional radiotherapy versus chemotherapy alone in primary metastatic nasopharyngeal carcinoma: A randomized, open-label, phase III trial. Ann. Oncol. 2019, 30, v449. [CrossRef]

16. Meyers, D.E.; Stukalin, I.; Vallerand, I.A.; Lewinson, R.T.; Suo, A.; Dean, M.; North, S.; Pabani, A.; Cheng, T.; Heng, D.Y.; et al. The Lung Immune Prognostic Index Discriminates Survival Outcomes in Patients with Solid Tumors Treated with Immune Checkpoint Inhibitors. Cancers 2019, 11, 1713. [CrossRef]

17. Hanahan, D.; Weinberg, R.A. Hallmarks of Cancer: The Next Generation. Cell 2011, 144, 646-674. [CrossRef] [PubMed]

18. Diakos, C.; Charles, K.A.; McMillan, D.; Clarke, S.J. Cancer-related inflammation and treatment effectiveness. Lancet Oncol. 2014, 15, e493-e503. [CrossRef]

19. Nebot-Bral, L.; Brandao, D.; Verlingue, L.; Rouleau, E.; Caron, O.; Despras, E.; El-Dakdoukibe, Y.; Champiat, S.; Aoufouchi, S.; Leary, A.; et al. Hypermutated tumours in the era of immuno-therapy: The paradigm of personalised medicine. Eur. J. Cancer Oxf. Engl. 1990 2017, 84, 290-303.

20. Atretkhany, K.-S.; Drutskaya, M.; Nedospasov, S.; Grivennikov, S.; Kuprash, D. Chemokines, cytokines and exosomes help tumors to shape inflammatory microenvironment. Pharmacol. Ther. 2016, 168, 98-112. [CrossRef] [PubMed]

21. Moon, Y.W.; Hajjar, J.; Hwu, P.; Naing, A. Targeting the indoleamine 2,3-dioxygenase pathway in cancer. J. Immunother. Cancer 2015, 3, 51. [CrossRef]

22. Tseng-Rogenski, S.S.; Hamaya, Y.; Choi, D.Y.; Carethers, J.M. Interleukin 6 Alters Localization of hMSH3, Leading to DNA Mismatch Repair Defects in Colorectal Cancer Cells. Gastroenterology 2015, 148, 579-589. [CrossRef] [PubMed] 
23. Blanc-Durand, F.; Rubio, X.M.; Auclin, E.; Ponce-Aix, S.; Castro, R.L.; Nadal, E.; Planchard, D.; Routy, B.; Hendriks, L.; Sullivan, I.; et al. FP07.06 Lung Immune Prognostic Index (LIPI) in Advanced NSCLC Patients Treated with Immunotherapy, Chemotherapy and both Combined Upfront. J. Thorac. Oncol. 2021, 16, S205-S206. [CrossRef]

24. Sorich, M.J.; Rowland, A.; Karapetis, C.; Hopkins, A. Evaluation of the Lung Immune Prognostic Index for Prediction of Survival and Response in Patients Treated With Atezolizumab for NSCLC: Pooled Analysis of Clinical Trials. J. Thorac. Oncol. 2019, 14, 1440-1446. [CrossRef] [PubMed]

25. Hopkins, A.; Kichenadasse, G.; Abuhelwa, A.; McKinnon, R.; Rowland, A.; Sorich, M. Value of the Lung Immune Prognostic Index in Patients with Non-Small Cell Lung Cancer Initiating First-Line Atezolizumab Combination Therapy: Subgroup Analysis of the IMPOWER150 Trial. Cancers 2021, 13, 1176. [CrossRef] [PubMed]

26. Ferrara, R.; Mezquita, L.; Texier, M.; Lahmar, J.; Audigier-Valette, C.; Tessonnier, L.; Mazieres, J.; Zalcman, G.; Brosseau, S.; Le Moulec, S.; et al. Hyperprogressive Disease in Patients With Advanced Non-Small Cell Lung Cancer Treated With PD-1/PD-L1 Inhibitors or With Single-Agent Chemotherapy. JAMA Oncol. 2018, 4, 1543-1552. [CrossRef] [PubMed]

27. Ferrara, R.; Mezquita, L.; Texier, M.; Lahmar, J.; Audigier-Valette, C.; Tessonnier, L.; Mazieres, J.; Zalcman, G.; Brosseau, S.; Le Moulec, S.; et al. Comparison of Fast-Progression, Hyperprogressive Disease, and Early Deaths in Advanced Non-Small-Cell Lung Cancer Treated With PD-1/PD-L1 Inhibitors or Chemotherapy. JCO Precis. Oncol. 2020, 829-840. [CrossRef] 